\title{
Comparison of endovascular treatment for middle cerebral artery aneurysm with a low-profile visualized intraluminal support stent or pipeline embolization device
}

\author{
PENG LU, YE ZHANG，HUANJIANG NIU and YIRONG WANG \\ Department of Neurosurgery, Sir Run Run Shaw Hospital, Zhejiang University School of Medicine, \\ Hangzhou, Zhejiang 310016, P.R. China
}

Received September 9, 2018; Accepted April 12, 2019

DOI: $10.3892 /$ etm.2019.7775

\begin{abstract}
The aim of the present study was to evaluate the safety and effectiveness of low-profile visualized intraluminal support (LVIS) stent and the pipeline embolization device (PED) for middle cerebral artery (MCA) aneurysm treatment. Data of patients with MCA aneurysms who received endovascular treatment with LVIS stent or PED added to the hospital's database between August 2016 and March 2018 were retrospectively collected, and the clinical results and angiographic outcomes were evaluated. A total of 43 patients were included in the study, of whom 23 received LVIS stents and 20 received PED. The rate of complete occlusion was similar in the two groups at 6 months post-treatment (90.9 vs. 88.9\%; $\mathrm{P}=0.832)$. Peri-operative complications were more frequent in the PED group; however, the LVIS group had more ischemic symptoms during the long-term follow-up. A larger aneurysm size $(\mathrm{P}=0.032)$ was associated with recanalization in the two groups. In conclusion, the LVIS stent and PED had acceptable rates of complete occlusion and aneurysm size was an independent predictor for recanalization. LVIS is more effective during the peri-operative period, while PED appears to have higher long-term safety.
\end{abstract}

Correspondence to: Professor Peng Lu, Department of Neurosurgery, Sir Run Run Shaw Hospital, Zhejiang University School of Medicine, 3 East Qingchun Road, Hangzhou, Zhejiang 310016, P.R. China

E-mail: doclupeng@zju.edu.cn

Abbreviations: MCA, middle cerebral artery; LVIS, low-profile visualized intraluminal support; EVT, endovascular treatment; PED, pipeline embolization device; MRA, magnetic resonance angiography; $\mathrm{SAH}$, subarachnoid hemorrhage

Key words: middle cerebral artery aneurysm, low-profile visualized intraluminal support, retrospective study, pipeline embolization device

\section{Introduction}

The middle cerebral artery (MCA) is a common location for cerebral intracranial aneurysms (1). MCA aneurysms are a leading cause of ischemic stroke and subarachnoid hemorrhage (SAH), either of which has a high mortality rate (2). MCA aneurysms also increase the risk of vascular injury, as they incorporate with acute-angled efferent branch vessels. Endovascular treatment is a widely accepted technique for treating of MCA aneurysms due to low morbidity rates as well as high efficacy $(3,4)$. At present, there are several different widely used clinical procedures, including reconstructive approaches using stents alone or in conjunction with coils $(5,6)$.

The most widely used stents include the Neuroform system (Stryker) (7,8), the Enterprise Vascular Reconstruction Device (DePuy Synthes; Johnson \& Johnson Medical Devices Companies) (9-11), Leo stent device (Balt Extrusion) $(12,13)$ and Low-profile visualized intraluminal support (LVIS) stent (Medtronic plc). The LVIS stent is the most frequently used approach for treating wide-necked and other types of complex cerebral aneurysm (14). It is a self-expanding, single-wire braid device with a nanoscale cell structure $(0.9 \mathrm{~mm})$ and higher metal coverage (23\%) compared to Neuroform (11\%) and Enterprise (10\%) (15). LVIS also provides good physical support for the regeneration of vascular endothelial cells. Compared with blood flow-guiding devices, the LVIS stent has a relatively small metal coverage, which reduces the risk of ischemic events (16). There is also a small LVIS stent called LVIS Junior (LVIS Jr) stent device, which may be deployed through a 0.017-inch luminal micro-catheter $(17,18)$.

Flow-diverting stents (FDSs) offer a more physiologic approach to MCA aneurysm treatment and have decreased porosity and higher metal coverage (19). FDSs are widely used for treating intracranial aneurysms that are not eligible for standard coiling treatment, and facilitate neointimal regrowth and remodeling of the arterial wall (20). The pipeline embolization device (PED) is the most commonly used FDS. It is a low-porosity, self-expanding, micro-catheter-delivered stent that contains 48-individual-mesh cobalt chromium and platinum strands. The metal coverage rate may reach $30-35 \%$, and the low-porosity structure alters the hemodynamics of the parent artery and reduces wall shear stress and promotes intraluminal emboli formation (21-23). 
The LVIS and the PED may be used for MCA aneurysm treatment; however, the differences between the safety and reliability of these two techniques remain elusive. Given this, a deeper understanding of angiographic outcomes following LVIS and PED is required.

In the present study, clinical and angiographic outcomes for 43 patients with MCA aneurysm treated with LVIS stent or PED were retrospectively compared and analyzed. Factors influencing stent recanalization were also examined. Furthermore, differences in flow reduction effects between LVIS stents and PED were assessed. The present study provides a comparison between LVIS and PED that may provide guidance regarding stent selection or the prediction of recanalization.

\section{Materials and methods}

Patient selection. The present study had a retrospective, comparative observational design. The data of patients that had been treated with either LVIS stent or PED were retrospectively collected from the database of Sir Run Run Shaw Hospital (Hangzhou, China), with records entered between August 2016 and March 2018 considered. The surgery reports, medical chart and radiologic images were collected and analyzed by four senior trained neuro-interventionists. All patients received conventional digital subtraction angiography (DSA) of the MCA. The aneurysm morphology and diameter of the parent artery were recorded. The analyzed data included aneurysm characteristics, peri-operative complications and treatment follow-up information.

Inclusion/exclusion criteria. All patients received a cerebral angiography to confirm the diagnosis and aneurysms located in the trunk of the M1 or M2 segment, or bifurcation of the MCA, were enrolled in the present study. All non-fatal MCA aneurysms underwent LVIS stent or PED procedures. The exclusion criteria were as follows: i) Small diameter ( $\leq 1.0 \mathrm{~mm})$ of parent artery or branch; ii) aneurysm was located in other vessels of the brain; ii) aneurysm ruptured prior to surgery. All patients who met the inclusion criteria were included in the study.

Endovascular treatment procedure. All patients were treated with anti-platelet therapy, including clopidogrel (75 mg/day) and aspirin (100 mg/day) for three to five days prior to the procedure. The procedures were performed under general anesthesia. Heparin was intravenously infused at an initial dose of 3,000 IU after the femoral sheath was placed. During the endovascular procedure, heparin was continuously infused at $1,000 \mathrm{IU} / \mathrm{h}$ to maintain heparinization. The working projection was determined by 3D rotational angiography (Siemens). Stent sizes were selected based on the largest diameter of the parent artery and the length of the aneurysm. After the stent was placed and the procedure was finished, patients were given dual anti-platelet therapy for at least six weeks and aspirin for a minimum of 6 months.

Aneurysm and vascular morphology definition. Rotational and conventional intra-arterial DSA were performed for $3 \mathrm{D}$ reconstruction in all patients. Aneurysms were defined based on their size and diameter, and characterized as large
(10-25 mm), small $(3-10 \mathrm{~mm})$ and tiny $(<3 \mathrm{~mm})$. Aneurysms with a fundus-to-neck ratio of $<2 \mathrm{~mm}$ or neck diameter of $>4 \mathrm{~mm}$ were defined as wide-necked aneurysms. The number of aneurysms was calculated and measured for each patient. The angle changes of vessels were also measured prior to and after surgery using the method described by Songsaeng et al (24). All data were reviewed and recorded by two independent neuro-interventionists.

Angiographic and clinical follow-up. On the basis of a review of clinical charts, delayed complications were also noted. The procedure-associated complications included thromboembolism, headache or dizziness, and intraprocedural rupture. The results of aneurysm occlusion were estimated based on the Raymond scale (RS) (25). Clinical outcome was assessed using the modified Rankin Scale (mRS) score (26) to evaluate the neurological status at discharge and during follow-up. The $\mathrm{mRS}$ scores were assessed by two independent neuro-interventionists at the time of follow-up visits. The angiographic follow-up was performed using magnetic resonance angiography (MRA) at 3 and 6 months using DSA. Based on the follow-up results of DSA, the stented arteries were classified as stable, or with progressive occlusion or recanalization.

Statistical analysis. SPSS (version 21.0; IBM Corp.) was used to analyze the data. Values are expressed as the mean \pm standard deviation or as number and percentage. Student's t-test or the Mann-Whitney U-test were used for continuous variables and the $\chi^{2}$ test was used for counting variables. Univariate logistic regression analysis was used to identify the predictors of procedural complications. $\mathrm{P}<0.05$ was considered to indicate a statistically significant difference.

\section{Results}

Characteristics of patients. A total of 43 patients with MCA aneurysms were included in the present study. The cohort included 26 males and 15 females with a mean age of 56.2 years (range, 37-79 years); 23 patients were treated with LVIS and 20 patients with PED. The clinicopathological and demographic characteristics of the patients are presented in Table I. In the LVIS group, the mean age was 58.78 years (range, 46-79 years), while in the PED group, the mean age was 52.56 years (range, 37-75 years). Risk factors included hypertension, hyperlipidemia, diabetes mellitus, cerebral ischemic comorbidities, cardiac comorbidities, smoking and alcohol abuse. There were no significant differences between the LVIS group and PED group in terms of age, sex or risk factors.

Characteristics of aneurysms. The characteristics of the aneurisms are provided in Table II. In the 43 patients, 60 aneurysms were identified, all of which were saccular. In the LVIS group, aneurysms had a mean diameter of 7.0 $\mathrm{mm}$ (range, 5.5-7.8 mm) and a mean neck size of $3.2 \mathrm{~mm}$ (range, 2.1-4.0 $\mathrm{mm}$ ). In the PED group, aneurysms had a mean diameter of $8.3 \mathrm{~mm}$ (range, 6.3-8.5 mm) and a mean neck size of $3.7 \mathrm{~mm}$ (range, 2.8-4.1 mm). In the LVIS group, 14 aneurysms were located in the MCA bifurcation, 10 in the M1 segment and 8 in the M2 segment. In the PED group, there were 9 aneurysms in the MCA bifurcation, 9 in the M1 segment and 10 in the M2 segment. 
Table I. Baseline characteristics of the patients.

\begin{tabular}{lccc}
\hline Characteristic & LVIS group $(\mathrm{n}=23)$ & PED group $(\mathrm{n}=20)$ & P-value \\
\hline Age (years) & $58.78 \pm 7.8$ & $52.56 \pm 6.3$ & 0.391 \\
Male gender & $15(65.2)$ & $11(55)$ & 0.494 \\
Body mass index $\left(\mathrm{kg} / \mathrm{m}^{2}\right)$ & $25.52 \pm 2.8$ & $24.8 \pm 3.4$ & 0.450 \\
Smoking & $7(30.4)$ & $9(45.0)$ & 0.324 \\
Alcohol abuse & $2(8.7)$ & $5(25.0)$ & 0.149 \\
Hypertension & $7(30.4)$ & $5(25.0)$ & 0.692 \\
Diabetes mellitus & $2(8.7)$ & $1(5.0)$ & 0.635 \\
Hyperlipidemia & $3(13.0)$ & $4(20.0)$ & 0.538 \\
Cerebral ischemic symptoms & $1(4.3)$ & $2(10.0)$ & 0.468 \\
Cardiac symptoms & $2(8.9)$ & $1(5.0)$ & 0.635 \\
\hline
\end{tabular}

Values are expressed as the mean \pm standard deviation or $\mathrm{n}(\%)$. LVIS, low-profile visualized intraluminal support; PED, pipeline embolization device.

There was no procedure-associated mortality in either group. Among the 23 patients who received LVIS stent, artery occlusion based on instant angiographic results was scored as RS1 in 10 aneurysms (43.5\%), RS2 in 9 aneurysms (39.1\%) and RS3 in 4 (17.4\%). In the PED group, occlusion was scored as RS1 in 9 (45.0\%), RS2 in 6 (30.0\%) and RS3 in 3 aneurisms (15.0\%; Table II).

Anatomical follow-up outcome. The anatomical follow-up outcomes are presented in Table III. The 23 patients in the LVIS group underwent MRA follow-up every 3 months for a mean duration of 7.8 months (range, 6.0-8.0 months) following endovascular treatment. Furthermore, 18 patients in the PED group underwent MRA follow-up every 3 months for a mean total of 7.5 months (range, 5.8-9.0 months). The patients in the two groups had stable aneurysm embolization at the end of the follow-up. A total of 22 patients $(95.7 \%)$ in the LVIS group and 18 patients (90\%) in the PED group completed the 6-month DSA follow-up. One patient in the LVIS group declined the DSA follow-up and two patients in the PED group were excluded due to their poor clinical condition. The complete occlusion rate (stable + progressive occlusion) in the PED group was $90.9 \%$, while it was $88.9 \%$ in the LVIS group, and there was no significant difference between the two groups at the end of the follow-up ( $\mathrm{P}=0.832)$. In the LVIS group, two cases of recanalization were encountered and there were another two in the PED group. The recanalization rate was not significantly different between the two groups $(\mathrm{P}=0.832)$.

The factors associated with recanalization of the aneurysm on follow-up were analyzed using univariate logistic regression analysis (Table IV). The results indicated that the aneurysm size was significantly associated with recanalization (OR, 0.923; 95\% CI: 0.835-0.997; $\mathrm{P}=0.032$ ) and is therefore an independent predictor for peri-operative complications. Other factors, including initial occlusion rate, drug resistance and anterior circulation aneurysm were not associated with recanalization and were not statistically significant $(\mathrm{P}>0.05)$.

Clinical peri-operative and follow-up outcomes. Clinical peri-operative and follow-up outcomes are provided in
Table V. In terms of peri-operative complications, parent artery occlusion was observed in 4 patients in the PED group, and one patient had cerebral vasospasm in the LVIS group. The other procedures were completed without any complications and all of the patients were discharged in an overall stable condition. A total of 41 patients attended the first 3-month clinical follow-up. One patient in the PED group died during the follow-up period due to complications from congestive heart failure and one patient died due to intracerebral hemorrhage in the LVIS group. This may be explained by the lack of dual (aspirin and clopidogrel) anti-platelet treatment in the LVIS group. A total of 4 patients (18.2\%) in the LVIS group suffered from cerebral ischemic symptoms at end of follow-up. Of these, one patient experienced transient ischemic attacks and the other three patients experienced permanent ischemia, which occurred 4 months after discharge. In the PED group, only one patient $(5.2 \%)$ experienced ischemic symptoms, and this rate was lower than that in the LVIS group $(\mathrm{P}<0.05)$. At the 6-month follow-up, most patients $(n=40)$ were stable and asymptomatic. One patient in the LVIS group complained about transient paresthesia at 5 months following the procedure; however, the follow-up DSA revealed no intrastent stenosis. There were no significant differences between the mRS scores of the two groups at the time of discharge or over the whole follow-up period.

The mortality rate at the 6-month follow up was $4.3 \%$ in the LVIS group and $0 \%$ in the PED group. The overall permanent neurological morbidity rate was $13.6 \%$ in the LVIS group and $0.0 \%$ in the PED group.

\section{Discussion}

MCA aneurysms frequently occur in primary MCA bifurcation or trifurcation and are a common cause of numerous life-threatening diseases, including SAH. Since the publication of the results of the International Subarachnoid Aneurysm Trial (27), endovascular treatment has become widely used. However, this treatment remains technically challenging and the long-term stability outcomes remain controversial. This study investigated patients who received LVIS stents or PED to 
Table II. Characteristics of MCA aneurysms.

\begin{tabular}{lcc}
\hline Characteristic & LVIS group & PED group \\
\hline Number of aneurysms & $32(22)$ & $28(18)$ \\
Location n (\%) & & $9(32.1)$ \\
MCA bifurcation & $14(43.8)$ & $9(32.1)$ \\
M1 segment & $10(31.2)$ & $10(35.8)$ \\
M2 segment & $8(25.0)$ & $8.3 \pm 5.6$ \\
Diameter (mm) & $7.0 \pm 4.1$ & $3.7 \pm 0.6$ \\
Neck width (mm) & $3.2 \pm 0.9$ & $3.9 \pm 1.1$ \\
Parent artery diameter (mm) & $3.5 \pm 0.9$ & $10(35.7)$ \\
Number of stents & & $15(53.6)$ \\
1 & $15(46.9)$ & $3(10.7)$ \\
2 & $16(50.0)$ & 0.305 \\
$\geq 3$ & $1(3.1)$ & 0.556 \\
Initial RS score & & $9(45.0)$ \\
RS1 & $10(43.5)$ & $6(30.0)$ \\
RS2 & $9(39.1)$ & $3(25.0)$ \\
RS3 & $4(17.4)$ & 0.809 \\
\hline
\end{tabular}

Values are expressed as the mean \pm standard deviation or $\mathrm{n}(\%)$. LVIS, low profile visualized intraluminal support; PED, pipeline embolization device; MCA, middle cerebral artery; RS, Raymond scale.

Table III. Follow-up angiographic results.

\begin{tabular}{lcc}
\hline Parameter & LVIS group & PED group \\
\hline Number of patients followed-up & 22 & 18 \\
Angiographic follow-up time (months) & $7.8 \pm 1.2$ & $7.5 \pm 1.4$ \\
Angiographic outcome & & \\
Stable & $15(68.2)$ & $13(72.2)$ \\
Progressive occlusion & $5(22.7)$ & $3(16.7)$ \\
Recanalization & $2(9.1)$ & $2(11.1)$ \\
Stable + progressive occlusion & $20(90.9)$ & $16(88.9)$ \\
RS score at last follow-up & & $12(66.7)$ \\
RS1 & $16(72.7)$ & $5(27.8)$ \\
RS2 & $4(18.2)$ & $1(5.5)$ \\
RS3 & $2(9.1)$ & 0.677 \\
\hline
\end{tabular}

Values are expressed as the mean \pm standard deviation or $\mathrm{n}(\%)$. LVIS, low-profile visualized intraluminal support; PED, pipeline embolization device; RS, Raymond scale.

treat MCA aneurysms and aimed to determine the long-term clinical safety and efficacy of these two techniques.

In the 1990s, Turjman et al (28) first used a stent-like endoprosthesis for the treatment of carotid artery aneurysms in an animal model. Since then, numerous different types of intracranial stent have been developed and used, including the Neuroform stent, Enterprise, Leo Baby, Solitaire stents as well as the LVIS stent (29-31). Compared with other stents, the LVIS stent was determined to be safer and more effective in treating intracranial aneurysms, particularly for wide-necked and dissecting aneurysms. This may be explained by its excellent conformability, apposition to the vessel wall and stability (32). A specific subtype of the LVIS stent called the LVIS Jr is a smaller device and may be delivered through micro-catheters (33). Recently, a systematic review from Zhang et al (34), including 384 patients with 390 aneurysms, revealed that LVIS is feasible, safe and effective for treating intracranial aneurysms, particularly in the short term. A serial case report also reported that the LVIS Jr device has a lower risk of thromboembolic complications compared to other devices (14). In a single-center trail, 97 patients with intracranial saccular aneurysms received a 
Table IV. Predictors of peri-operative complications.

\begin{tabular}{lccr}
\hline & \multicolumn{2}{c}{ Univariate analysis } & \\
\cline { 2 - 3 } Parameter & OR & $95 \%$ CI & P-value \\
\hline Aneurysm size (>10 mm) & 0.923 & $0.835-0.997$ & 0.032 \\
Initial occlusion rate (complete vs. incomplete) & 0.938 & $0.357-2.654$ & 0.825 \\
Drug resistance (present vs. absent) & 1.589 & $0.988-4.325$ & 0.135 \\
Anterior circulation aneurysm (present vs. absent) & 1.000 & $0.316-2.655$ & 1.000 \\
\hline
\end{tabular}

LVIS, low-profile visualized intraluminal support; OR, odds ratio; CI, confidence interval.

Table V. Clinical peri-operative and follow-up outcome.

\begin{tabular}{lrrr}
\hline Results & LVIS group & PED group & P-value \\
\hline Peri-operative complications & & & 0.488 \\
None & $22(95.7)$ & $16(80.0)$ & 0.024 \\
Parent artery occlusion & $0(0.0)$ & $4(20.0)$ & 0.345 \\
Cerebral vasospasm & $1(4.3)$ & $0(0.0)$ & 0.468 \\
mRS score 0-2 & & & 0.677 \\
At discharge & $22(95.7)$ & $18(90.0)$ & 0.057 \\
During clinical follow-up & $16(72.7)$ & $0(0.0)$ & 0.085 \\
Neurological morbidity & $3(13.6)$ & $1(5.2)$ & 0.603 \\
Cerebral ischemic symptoms & $4(18.2)$ & $0(0.0)$ &
\end{tabular}

LVIS, low-profile visualized intraluminal support; PED, pipeline embolization device; mRS, modified Rankin Scale.

LVIS stent and none of them had any target aneurysm recurrence and no mortalities occurred (35). For other types of aneurysm, including blood blister-like aneurysms, the LVIS stent is associated with a lesser risk of aneurysm recurrence compared to non-LVIS stents (36). Compared with enterprise stent-assisted coiling, the LVIS achieved a similar complete and near-complete aneurism embolization rate (37). In the present study, the patients who received the LVIS stent had fewer peri-operative complications compared with those in the PED group, suggesting that the LVIS stent is safer in the short term, which is consistent with Zhang et al (34) meta-analysis study. However, patients in the LVIS group experienced more ischemic symptoms during long-term follow-up compared with those in the PED group, suggesting the LVIS stent may cause microthrombosis.

The PED is a flow diverter device that may be placed across the aneurysm neck to disrupt and divert the flow to the point of stagnation. This results in the remodeling of the arterial wall, reducing the rate of rupture and lowering the recanalization rate (38). The first clinical use of a PED was reported in North America in 2008 (39), where PEDs are made of 25\% platinum and $75 \%$ nickel-cobalt chromium, with a high metal coverage (30-35\%). The diameter of this device ranges from 2.5 to $5 \mathrm{~mm}$. Originally, the PED was indicated for fusiform or wide-necked aneurysms; however, this device is used to treat other types of aneurysm, including anterior cerebral artery aneurysms, recurrent aneurysms and dissect aneurysms (40-42). A meta-analysis including 200 patients and 215 aneurysms treated with PED revealed that the complete obliteration rate of PED for giant intracranial aneurysms was $\sim 60 \%$ (43). Another systematic review focusing on complications and mortality rate after PED treatment indicated no significant difference between the PED and the silk flow-diverter device (44). Furthermore, Rouchaud et al (45) reported that in a study of 265 patients, PED had a significantly higher long-term aneurysm occlusion rate compared with that of other endovascular techniques; however, other outcomes, including mortality and hemorrhage, were not significantly different. However, when treating acutely ruptured aneurysms, the application of PED remains limited (45). In the present study, although patients in the PED group had more peri-operative complications, the recanalization rate was lower compared with that in the LVIS stent group. In the long term, PED treatment appears to be safer due to the lower rate of ischemic symptoms.

Endovascular treatment is now the most commonly used treatment option for MCA aneurysms over craniotomy clipping. MCA aneurysms may be treated using either a LVIS stent or PED (46). For instance, Feng et al (17) reported on the treatment of 18 MCA aneurysms with the LVIS Jr; the rate of complete occlusion was $44.4 \%$ without any technical issues and it was suggested that the LVIS Jr stent is safer and more effective for treating the wide-necked MCA aneurysms compared 
to other treatment approaches. Yavuz et al (47) reported on 25 aneurysms located at the MCA bifurcation that were treated with PED and no deaths occurred, suggesting it is a safe and effective treatment alternative for wide-neck MCA aneurysms. PEDs have more flexibility and better transport properties and therefore offer a promising treatment for wide-necked MCA aneurysms. To the best of our knowledge, a comparison of outcomes between these different techniques has not been previously performed. In the present study, information from 43 patients with MCA aneurysms added to the database between August 2016 and March 2018 was retrospectively collected and reviewed. Patients either received an LVIS stent or a PED. The results suggested that the rate of complete occlusion was similar in the two groups at 6 months post-procedure $(90.9$ vs. $88.9 \%$; $\mathrm{P}=0.832$ ). Peri-operative complications were more common in the PED group; however, more ischemic symptoms were encountered in the LVIS group during long-term follow-up. The recanalization rate was lower in the PED group. A larger aneurysm size was associated with recanalization in the two groups. The present results suggested that the LVIS and the PED had acceptable rates of complete occlusion in the treatment of MCA aneurysms, with LVIS being more effective in the peri-operative period and PED being safer in the long term.

There are certain limitations of the present study that should be considered. First, it was a retrospective study with a relatively small sample size. Therefore, studies with larger sample sizes should be performed. Furthermore, all data were collected in a single center; thus, a selection bias may have prevailed. In addition, the follow-up period was relatively short, and therefore, the present results may not allow for any conclusions regarding long-term outcomes. Further studies are required with a larger sample size and a longer follow-up period.

The present study assessed 43 patients with 60 aneurysms. In the LVIS and the PED group, the complete occlusion rate was acceptable and there were no significant differences. The PED group was indicated to have a lower rate of recanalization. LVIS proved to be more effective during the peri-operative period, while PED appears safer in the long term. Finally, aneurysm size was identified to be an independent predictor of recanalization.

\section{Acknowledgements}

Not applicable.

\section{Funding}

No funding was received.

\section{Availability of data and materials}

The datasets used and/or analyzed during the present study are available from the corresponding author on reasonable request.

\section{Authors' contributions}

PL and YZ performed the experiments of the current study. YZ, HN and YW prepared and analyzed experimental data. YW designed the experiments. All authors read and approved the final version of the manuscript.

\section{Ethical approval and consent to participate}

The study protocol was approved by the Ethics Committee of Sir Run Run Shaw Hospital (Hangzhou, China).

\section{Patient consent for publication}

Not applicable.

\section{Competing interests}

The authors declare that they have no financial or other types of competing interest.

\section{References}

1. Zaidat OO, Castonguay AC, Teleb MS, Asif K, Gheith A, Southwood C, Pollock G and Lynch JR: Middle cerebral artery aneurysm endovascular and surgical therapies: Comprehensive literature review and local experience. Neurosurg Clin N Am 25: 455-469, 2014.

2. Moroi J, Hadeishi H, Suzuki A and Yasui N: Morbidity and mortality from surgical treatment of unruptured cerebral aneurysms at research institute for brain and blood Vessels-Akita. Neurosurgery 56: 224-231, 2005.

3. Pierot L and Wakhloo AK: Endovascular treatment of intracranial aneurysms: Current status. Stroke 44: 2046-2054, 2013.

4. Shen X, Xu T, Ding X, Wang W, Liu Z and Qin H: Multiple intracranial aneurysms: Endovascular treatment and complications. Interv Neuroradiol 20: 442-447, 2014.

5. Molyneux AJ, Birks J, Clarke A, Sneade M and Kerr RS: The durability of endovascular coiling versus neurosurgical clipping of ruptured cerebral aneurysms: 18 year follow-up of the UK cohort of the international subarachnoid aneurysm trial (ISAT). Lancet 385: 691-697, 2015.

6. Thomas AJ and Ogilvy CS: ISAT: Equipoise in treatment of ruptured cerebral aneurysms? Lancet 385: 666-668, 2015.

7. Benitez RP, Silva MT, Klem J, Veznedaroglu E and Rosenwasser RH: Endovascular occlusion of wide-necked aneurysms with a new intracranial microstent (Neuroform) and detachable coils. Neurosurgery 54: 1359-1367, 2004.

8. Fiorella D, Albuquerque FC, Deshmukh VR and McDougall CG: Usefulness of the Neuroform stent for the treatment of cerebral aneurysms: Results at initial (3-6-mo) follow-up. Neurosurgery 56: 1191-1202, 2005.

9. Mocco J, Snyder KV, Albuquerque FC, Bendok BR, Alan SB, Carpenter JS, Fiorella DJ, Hoh BL, Howington JU, Jankowitz BT, et al: Treatment of intracranial aneurysms with the Enterprise stent: A multicenter registry. J Neurosurg 110: 35-39, 2009.

10. Higashida RT, Halbach VV, Dowd CF, Juravsky L and Meagher S: Initial clinical experience with a new self-expanding nitinol stent for the treatment of intracranial cerebral aneurysms: The Cordis Enterprise stent. AJNR Am J Neuroradiol 26: 1751-1756, 2005.

11. Weber W, Bendszus M, Kis B, Boulanger T, Solymosi L and Kuhne D: A new self-expanding nitinol stent (Enterprise) for the treatment of wide-necked intracranial aneurysms: Initial clinical and angiographic results in 31 aneurysms. Neuroradiology 49: 555-561, 2007.

12. Pumar JM, Blanco M, Vázquez F, Castiñeira JA, Guimaraens L and Garcia-Allut A: Preliminary experience with Leo self-expanding stent for the treatment of intracranial aneurysms. AJNR Am J Neuroradiol 26: 2573-2577, 2005.

13. Juszkat R, Nowak S, Smol S, Kociemba W, Blok T and Zarzecka A: Leo stent for endovascular treatment of broad-necked and fusiform intracranial aneurysms. Interv Neuroradiol 13: 255-269, 2007.

14. Behme D, Weber A, Kowoll A, Berlis A, Burke TH and Weber W: Low-profile Visualized Intraluminal Support device (LVIS Jr) as a novel tool in the treatment of wide-necked intracranial aneurysms: Initial experience in 32 cases. J Neurointerv Surg 7: 281-285, 2015.

15. Poncyljusz W, Bilinski P, Safranow K, Baron J, Zbroszczyk M, Jaworski M, Bereza S and Burke TH: The LVIS/LVIS Jr. Stents in the treatment of wide-neck intracranial aneurysms: Multicentre registry. J Neurointerv Surg 7: 524-529, 2015. 
16. Ge H, Lv X, Yang X, He H, Jin H and Li Y: LVIS stent versus enterprise stent for the treatment of unruptured intracranial aneurysms. World Neurosurg 91: 365-370, 2016.

17. Feng Z, Li Q, Zhao R, Zhang P, Chen L, Xu Y, Hong B, Zhao W Liu J and Huang Q: Endovascular treatment of middle cerebral artery aneurysm with the LVIS junior stent. J Stroke Cerebrovasc Dis 24: 1357-1362, 2015.

18. Alghamdi F, Mine B, Morais R, Scillia P and Lubicz B: Stent-assisted coiling of intracranial aneurysms located on smal vessels: Midterm results with the LVIS Junior stent in 40 patients with 43 aneurysms. Neuroradiology 58: 665-671, 2016.

19. Agarwal A, Gokhale S, Gupta J, Raju R, Nimjee S, Smith T and Britz GW: Use of pipeline flow diverting stents for wide neck intracranial aneurysms: A retrospective institutional review. Asian J Neurosurg 9: 3-6, 2014.

20. Chitale R, Gonzalez LF, Randazzo C, Dumont AS, Tjoumakaris S, Rosenwasser R, Chalouhi N, Gordon D and Jabbour P: Single center experience with pipeline stent: Feasibility, technique, and complications. Neurosurgery 71: 679-691, 2012.

21. Brinjikji W, Lanzino G, Cloft HJ, Siddiqui AH, Hanel RA and Kallmes DF: Platelet testing is associated with worse clinical outcomes for patients treated with the pipeline embolization device. AJNR Am J Neuroradiol 36: 2090-2095, 2015.

22. Daou B, Starke RM, Chalouhi N, Tjoumakaris S, Hasan D, Khoury J, Rosenwasser RH and Jabbour P: Pipeline embolization device in the treatment of recurrent previously stented cerebral aneurysms. AJNR Am J Neuroradiol 37: 849-855, 2016.

23. Brinjikji W, Lanzino G, Cloft HJ, Siddiqui AH and Kallmes DF: Risk factors for hemorrhagic complications following pipeline embolization device treatment of intracranial aneurysms: Results from the international retrospective study of the pipeline embolization device. AJNR Am J Neuroradiol 36: 2308-2313, 2015.

24. Songsaeng D, Geibprasert S, ter Brugge KG, Willinsky R, Tymianski $M$ and Krings T: Impact of individual intracranial arterial aneurysm morphology on initial obliteration and recurrence rates of endovascular treatments: A multivariate analysis J Neurosurg 114: 994-1002, 2011.

25. Raymond J and Roy D: Safety and efficacy of endovascular treatment of acutely ruptured aneurysms. Neurosurgery 41: 1235-1246, 1997.

26. Broderick JP, Adeoye O and Elm J: Evolution of the modified rankin scale and its use in future stroke trials. Stroke 48: 2007-2012, 2017.

27. Mascitelli JR, Cole T, Yoon S, Nakaji P, Albuquerque FC, McDougall CG, Zabramski JM, Lawton MT and Spetzler RF: External validation of the subarachnoid hemorrhage internationa trialists (SAHIT) predictive model using the barrow-ruptured aneurysm trial (BRAT) Cohort. Neurosurgery, Dec 19, 2018 (Epub ahead of print).

28. Turjman F, Acevedo G, Moll T, Duquesnel J, Eloy R and Sindou M: Treatment of experimental carotid aneurysms by endoprosthesis implantation: Preliminary report. Neurol Res 15 : 181-184, 1993.

29. Ferrell AS, Golshani K, Zomorodi A, Smith TP and Britz GW: Improved delivery of the Neuroform 3 stent: Technical note. J Neurointerv Surg 4: 287-290, 2012.

30. Prasad S, Su HC, Altay N and Tata J: Building disaster-resilient micro enterprises in the developing world. Disasters 39: 447-466, 2015.

31. Akmangit I, Aydin K, Sencer S, Topcuoglu OM, Topcuoglu ED, Daglioglu E, Barburoglu M and Arat A: Dual stenting using low-profile LEO baby stents for the endovascular management of challenging intracranial aneurysms. AJNR Am J Neuroradiol 36 : 323-329, 2015

32. Zhang M, Ouyang Y, Huang H, Jiang Y and Yu M: Application of LVIS stents in very small intracranial aneurysms. Zhong Nan Da Xue Xue Bao Yi Xue Ban 41: 821-825, 2016 (In Chinese).
33. Möhlenbruch M, Herweh C, Behrens L, Jestaedt L, Amiri H, Ringleb PA, Bendszus M and Pham M: The LVIS Jr. Microstent to assist coil embolization of wide-neck intracranial aneurysms: Clinical study to assess safety and efficacy. Neuroradiology 56 : 389-395, 2014.

34. Zhang X, Zhong J, Gao H, Xu F and Bambakidis NC: Endovascular treatment of intracranial aneurysms with the LVIS device: A systematic review. J Neurointerv Surg 9: 553-557, 2017.

35. Feng Z, Fang Y, Xu Y, Hong B, Zhao W, Liu J and Huang Q: The safety and efficacy of low profile visualized intraluminal support (LVIS) stents in assisting coil embolization of intracranial saccular aneurysms: A single center experience. J Neurointerv Surg 8: 1192-1196, 2016.

36. Zhu D, Fang Y, Yang P, Zhang P, Chen L, Xu Y, Hong B, Huang Q and Liu JM: Overlapped stenting combined with coiling for blood blister-like aneurysms: Comparison of low-profile visualized intraluminal support (LVIS) stent and Non-LVIS stent. World Neurosurg 104: 729-735, 2017.

37. Feng X, Qian Z, Liu P, Zhang B, Wang L, Guo E, Wen X, Xu W, Jiang C, Wu Z, et al: Comparison of recanalization and in-stent stenosis between the low-profile visualized intraluminal support stent and enterprise stent-assisted coiling for 254 intracranial aneurysms. World Neurosurg 109: e99-e104, 2018.

38. Alderazi YJ, Shastri D, Kass-Hout T, Prestigiacomo CJ and Gandhi CD: Flow diverters for intracranial aneurysms. Stroke Res Treat 2014: 415653, 2014.

39. Fiorella D, Woo HH, Albuquerque FC and Nelson PK: Definitive reconstruction of circumferential, fusiform intracranial aneurysms with the pipeline embolization device. Neurosurgery 62: 1115-1121, 2008

40. Chalouhi N, Tjoumakaris S, Phillips JL, Starke RM, Hasan D, Wu C, Zanaty M, Kung D, Gonzalez LF, Rosenwasser R and Jabbour P: A single pipeline embolization device is sufficient for treatment of intracranial aneurysms. AJNR Am J Neuroradiol 35: 1562-1566, 2014

41. Raz E, Shapiro M, Becske T,Zumofen DW, Tanweer O, Potts MB, Riina HA and Nelson PK: Anterior choroidal artery patency and clinical follow-up after coverage with the pipeline embolization device. AJNR Am J Neuroradiol 36: 937-942, 2015.

42. Chalouhi N, Chitale R, Starke RM, Jabbour P, Tjoumakaris S, Dumont AS, Rosenwasser RH and Gonzalez LF: Treatment of recurrent intracranial aneurysms with the pipeline embolization device. J Neurointerv Surg 6: 19-23, 2014.

43. $\mathrm{Lv} \mathrm{X}, \mathrm{Ge} \mathrm{H}, \mathrm{He} \mathrm{H}$, Jiang $\mathrm{C}$ and $\mathrm{Li} \mathrm{Y}$ : A systematic review of pipeline embolization device for giant intracranial aneurysms. Neurol India 65: 35-38, 2017.

44. Zhou G, Su M, Yin YL and Li MH: Complications associated with the use of flow-diverting devices for cerebral aneurysms: A systematic review and meta-analysis. Neurosurg Focus 42: E17, 2017.

45. Rouchaud A, Brinjikji W, Cloft HJ and Kallmes DF: Endovascular treatment of ruptured blister-like aneurysms: A systematic review and meta-analysis with focus on deconstructive versus reconstructive and flow-diverter treatments. AJNR Am J Neuroradiol 36: 2331-2339, 2015.

46. Darflinger RJ and Chao K: Using the barrel technique with the LVIS Jr (Low-profile Visualized Intraluminal Support) stent to treat a wide neck MCA bifurcation aneurysm. J Vasc Interv Neurol 8: 25-27, 2015

47. Yavuz K, Geyik S, Saatci I and Cekirge HS: Endovascular treatment of middle cerebral artery aneurysms with flow modification with the use of the pipeline embolization device. AJNR Am J Neuroradiol 35: 529-535, 2014.

This work is licensed under a Creative Commons Attribution-NonCommercial-NoDerivatives 4.0 International (CC BY-NC-ND 4.0) License. 